\title{
Ordinary Kriging Applied to Fluid Measurement
}

\author{
Mengyuan Wang1, a, *, Shi Liu ${ }^{1, b}$, Yubo Liang', c, and H.I. Schlaberg ${ }^{1, d}$ \\ ${ }^{1}$ School of control and computer engineering, North China Electric Power University, Beijing \\ 102206, China \\ amyWang@ncepu.edu.cn, ${ }^{b}$ liushi@ncepu.edu.cn, ${ }^{c}$ lyb941002@163.com, ${ }^{d}$ h.i.schlaberg@ieee.org
}

Keywords: ordinary Kriging, sensor optimization, flow pattern.

Abstract: The identification of flow pattern is a basic and important issue in multiphase systems. However, it is difficult to discern due to the complexity of two-phase flows. In this paper, ordinary Kriging(OK) is applied to reconstructed the two-phase flow patterns using some accurate data. Three typical flow patterns are preset to verify the effectiveness of the method and two quality assessments are used to verify it. Computational results demonstrate that two-phase flow patterns can be reconstructed successfully. The method is feasible for fluid measurement applications.

\section{Introduction}

Two-phase flows is commonly exist in many industrial processes such as chemical, nuclear and petroleum industries [1]. For the measurement of two-phase flow characteristics, process tomography (PT) is widely used [2]. The PT technique has the advantages of being a non-invasive measurement, having a fast response and being of low cost. However, due to it being ill-posed and non-linear, the accuracy of reconstructed PT images is low [3].

Kriging is the most frequently used spatial prediction technique. It has been widely used in geography [4]. It is different from classical modeling approaches in that they incorporate information on the geographic location of the sample points. The prediction map is calculated by a weighted average of nearby points. In this paper, OK is applied to fluid measurements. Two-phase flow patterns predicted by data points are explored in this paper.

\section{Method}

\subsection{Ordinary Kriging}

Ordinary Kriging is one of the most basic kriging methods [5]. It provides a prediction at an unobserved location of variable $\mathrm{z}$, based on the weighted average of adjacent observed sites. The theory can be briefly described by considering an intrinsic random function denoted by $\mathrm{z}\left(\mathrm{s}_{\mathrm{i}}\right)$, where $\left(s_{i}\right)$ are sample locations, $i=1,2, \ldots, n$. Using the ordinary Kriging predictor, an estimate of the weighted 
average at an unsampled location is defined by:

$$
\mathrm{Z}\left(s_{0}\right)=\sum_{i=1}^{n} \lambda_{i} z\left(s_{i}\right)
$$

Where: $\lambda$ are the weights corresponding to the observed data. The predictor provides an unbiased estimation by summing these weights to unity:

$$
\sum_{i=1}^{n} \lambda_{i}=1
$$

The weights are calculated from the matrix equation:

$$
C=A^{-1} b
$$

Where: A is a matrix of semi-variances between the observed points. $\mathrm{b}$ is a vector of estimated semi-variances between the data points and the points to be predicted. $\mathrm{C}$ are the weights.

\subsection{Quality Assessments}

In this paper, image quality prediction will be assessed by the relative error and the correlation coefficient, with

Relative error,

$$
\delta=\frac{\sum_{i=1}^{n}\left(\hat{z}_{i}-z_{i}\right)}{\sum_{i=1}^{n} z_{i}} \times 100 \%
$$

and Correlation coefficient,

$$
r=\frac{\sum_{i=1}^{n}\left(\hat{z}_{i}-\overline{\hat{z}}_{i}\right) \times\left(z_{i}-\bar{z}\right)}{\sqrt{\sum_{i=1}^{n}\left(\hat{z}_{i}-\overline{\hat{z}}_{i}\right)^{2} \sum_{i=1}^{n}\left(z_{i}-\bar{z}\right)^{2}}} \times 100 \%
$$

Where: $\hat{z}$ and $\mathrm{z}$ are the grayscale values of a pixel in the predicted image and the preset image respectively. A smaller relative error means the image suffers from lower noise effects. The image which is more similar to the preset one has a larger correlation coefficient.

\section{Simulation and Results}

To verify our method, three typical flow patterns are preset. They are the core flow, scatterd flow and sedimented flow. The image has a total of 6400 pixels. The number of data points used for reconstruction is 49 , i.e. $0.77 \%$ of the total pixels. The Point observations are distributed evenly. The results are shown in Fig.1 to Fig.3. The predicted map is very similar to the preset one. The two objects in the map is reconstructed successfully. The quality assessments of the three flow patterns is assessed by two statistics. As shown in Table 1. the map reconstructed by OK has a low relative error and is very similar to the preset one. 

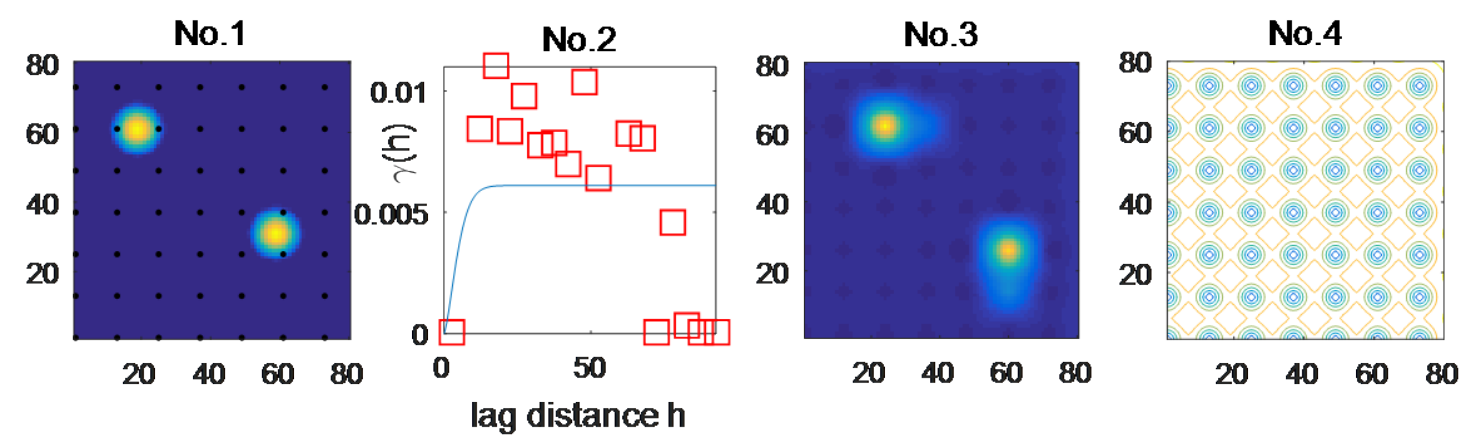

Fig. 1 Prediction results of Core Flow. No.1 shows the image with sampling locations. No. 2 shows the variogram. No.3 shows the kriging predictions. No.4 shows the kriging variance
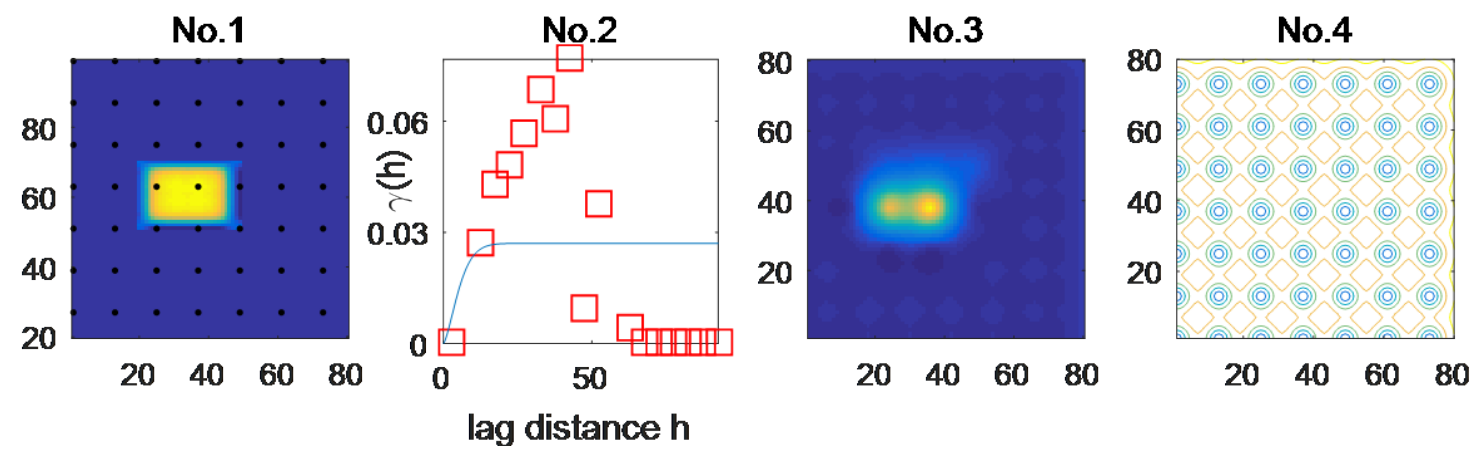

Fig. 2 Prediction results of Scattered Flow. No.1 shows the image with sampling locations. No.2 shows the variogram. No.3 shows the kriging predictions. No.4 shows the kriging variance
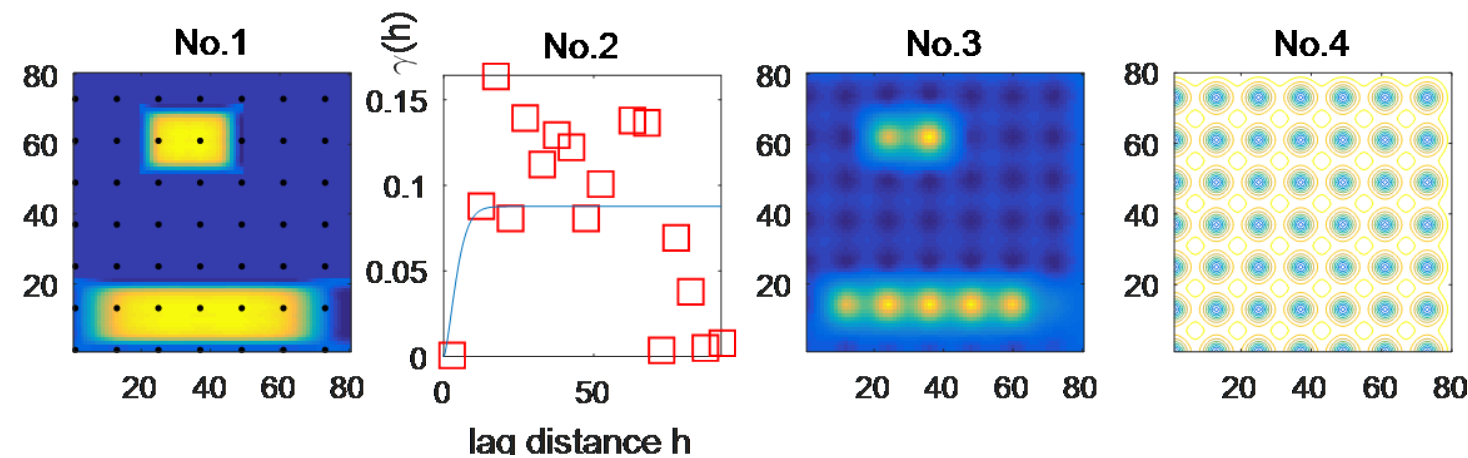

Fig. 3 Prediction results of Sedimented Flow. No.1 shows the image with sampling locations. No.2 shows the variogram. No.3 shows the kriging predictions. No.4 shows the kriging variance

Table 1 Quality Assessments

\begin{tabular}{cccc}
\hline Flow pattern & Core Flow & Scattered Flow & $\begin{array}{c}\text { Sedimented } \\
\text { Flow }\end{array}$ \\
\hline Relative error(\%) & 65.35 & 85.38 & 85.1 \\
Correlation error(\%) & 60.23 & 31.37 & 29.93 \\
\hline
\end{tabular}

\section{Conclusion}

Ordinary Kriging is used in this paper to rebuild the map of two-phase flow patterns. Three typical flow patterns are preset. The results show that OK can reconstruct maps with few data points. By calculating the relative error and the correlation error, this method is verified its effectiveness in fluid 
measurements.

\section{References}

[1] Polansky Jiri, Wang Mi. 2017. Proper Orthogonal Decomposition as a technique for identifying two-phase flow pattern based on electrical impedance tomography, Flow Measurement \& Instrumentation, Vol.53(Part A):126-132.

[2] Xiao Liu, Xiaoxin Wang, Hongli Hu, et al. 2015. An extreme learning machine combined with Landweber iteration algorithm for the inverse problem of electrical capacitance tomography, Flow Measurement and Instrumentation, 348-356.

[3] T York. 2001. Status of electrical tomography in industrial applications, Process Imaging for Automatic Control, 4188(10):608-619.

[4] J. Negreiros, M. Painho, F. Aguilar. 2010. Geographical Information Systems Principles of Ordinary Kriging Interpolator, Journal of Applied Sciences, Vol.10(No.11):852-867.

[5] Jafar Yasrebi, Mahboub Saffari, Hamed Fathi, et al. 2012. Evaluation and Comparison of Ordinary Kriging and Inverse Distance Weighting Methods for Prediction of Spatial Variability of Some Soil Chemical Parameters, Research Journal of Biological Sciences. 